\title{
Multi-electrode endovascular denervation at a new site of celiac artery for glycemic control in patients with type 2 diabetes mellitus: a promisingly primary result
}

\author{
Tao Pan \\ Vascular and Interventional Radiology \\ Qiong Wei \\ Southeast University Zhongda Hospital \\ Zhi Wang \\ Southeast University Zhongda Hospital \\ Qi Zhang \\ Southeast University Zhongda Hospital

\section{Yue Qian}

Nanjing Drum Tower Hospital: Nanjing University Medical School Affiliated Nanjing Drum Tower Hospital

Rui Li

Southeast University Zhongda Hospital

De-Chen Liu

Southeast University Zhongda Hospital

\section{Yao Wang}

Southeast University Zhongda Hospital

\section{Zi-Lin Sun}

Southeast University Zhongda Hospital

Ling Li

Southeast University Zhongda Hospital

Gao-Jun Teng ( $\sim$ gjteng@vip.sina.com )

Vascular and Interventional Radiology

Original investigation

Keywords: multi-electrode endovascular denervation, type 2 diabetes mellitus, celiac artery, safety, glycemic control, insulin resistance

Posted Date: March 31st, 2021 
DOl: https://doi.org/10.21203/rs.3.rs-357352/v1

License: (c) (1) This work is licensed under a Creative Commons Attribution 4.0 International License. Read Full License 


\section{Abstract}

Background: Although renal denervation (RDN) may theoretically be effective on type 2 diabetes mellitus (T2DM), most clinical data failed to demonstrate such a concept. We investigated the safety and primary effects of catheter-based endovascular denervation (EDN) at new sites of the celiac artery (CA), and the abdominal aorta around CA using a novel multi-electrode device on glycemic control in T2DM patients.

Methods: With a novel six-electrode catheter system, EDN was conducted in T2DM patients at new sites of CA and aorta between CA and superior mesenteric artery (SMA) in purpose of targeting on the postganglionic efferent nerves innervating the liver, islet, and other metabolic organs. The major inclusion criteria were T2DM patients diagnosed over 5 years, with hemoglobin A1c (HbA1c) over 7.5\%. The primary outcomes were evaluated by the safety, $\mathrm{HbA1c}$, homeostasis-model assessment of insulin resistance (HOMA-IR), fasting plasma glucose (FPG), 2-hour postprandial plasma glucose (2hPG), and oral glucose tolerance test (OGTT) based 3-hour C-peptide test. The antidiabetic medication, lifestyle, physical condition, blood pressure (BP), plasma norepinephrine, angiotensin II, liver biochemistry, and plasma lipids were also recorded.

Results: A total of 11 patients were included for analysis. The technical success was $100 \%$ and no severe treatment-related adverse events or major complications were observed. Both HbA1c and HOMA-IR significantly reduced at 6 months ( 9.9 vs. $8.0, P=0.005 ; 13.3$ vs. $6.0, P=0.016$, respectively). Decreases of FPG and 2hPG were observed (15.4 vs. $10.1 \mathrm{mmol} / \mathrm{L}, P<0.001 ; 17.9$ vs. $11.4 \mathrm{mmol} / \mathrm{L}, P=0.001$, respectively). OGTT based C-peptide test indicated improved beta-cell function ([AUC] 0.23 vs. 0.28 $\mathrm{pmol} / \mathrm{mL}, P=0.046)$. A reduction of daily insulin injection $(P=0.02)$ and improvement of liver function (ALT, $P=0.014$; GGT, $P=0.021$ ) were also observed without changes in lifestyle, physical conditions, and other metabolic measurements.

Conclusions: The 6-month analysis of this trial showed that EDN at new sites of CA and the abdominal aorta around celiac artery using the novel six-electrode catheter system elicits a clinically significant improvement in glycemic control and insulin resistance in patients with T2DM, with good tolerability.

Trial Registration Number NCT 04086043, dated 11/9/2019.

\section{Background}

Type 2 diabetes mellitus (T2DM) has caused huge health burden worldwide, with the global prevalence of chronic hyperglycemia or diabetes being approximately one in 11 among adults[1]. Standard managements of T2DM include diet control, engaging in physical activity, weight control, and pharmacologic approaches[2-4]. Unfortunately, the poor compliance with complex medical pharmacologic regimens[5] and the complex pathophysiology of T2DM lead to a major challenge in glycemic control despite a wide choice of pharmacological interventions[6]. 
Chronic elevation of activity of the sympathetic nervous system (SNS) has been identified to induce insulin resistance[7] and contributes to the development of T2DM[8] and metabolic syndrome[9]. Therefore, catheter-based renal denervation (RDN) has been introduced tentatively since it has been debated as a potentially effective intervention to treat hypertension and related metabolic disorders[1012]. However, the DREAMS-Study[13], investigating the effects of RDN on blood pressure (BP) and insulin sensitivity in patients with metabolic syndrome, neither showed a change in fasting glucose and insulin sensitivity, nor improved sympathetic activity, possibly attributes to insufficient denervation. Therefore, the role of RDN in treating T2DM remains controversial.

Anatomically, sympathetic nerves arrive at the islet and liver via splanchnic nerves originating from prevertebral celiac and superior mesenteric ganglia[14,15], and directly innervate them to control hormone secretion, glucose production, and metabolism[16]. Additionally, sympathetic nerves regulate the anti-inflammatory effect of spleen[17] and the metabolism of adipose tissue[18, 19], both of which are involved, positively or oppositely, to the pathophysiology of T2DM. Experimental evidence demonstrates that surgical sympathetic denervation of the common hepatic artery improves glucose tolerance and enhances postprandial glucose clearance[20]. Clinical researches to date, however, have not focused on catheter-based denervation of other metabolism-related organs. Thus, we hypothesized that targeting the metabolism-related organs directly may have a more straightforward influence than RDN through directly turning down the sympathetic output to these organs.

Therefore, we innovatively applied a minimally invasive, catheter-based endovascular denervation (EDN) procedure at new sites of CA and aorta between CA and SMA by using a novel six-electrode catheter system to target the postganglionic nerves originating from celiac and superior mesenteric ganglia instead of renal afferent and efferent nerves, in order to treat T2DM. The novel six-electrode catheter system was designed to solve the problem of inadequate ablation by using single-electrode protocol[21, 22]. This technique was proved to be feasible and safe in relieving cancer pain in our former research[23]. However, it is worth studying whether EDN may provide a direct and effective approach to improve glucose metabolism by mediating the sympathetic nerve signaling sent to metabolism-related organs and then offer a new therapeutic method for T2DM. Here we conducted a first-in-human clinical study of EDN in patients with T2DM. We report on safety, tolerability, and effectiveness from the 6-month analysis.

\section{Methods}

\section{Study Design}

This was a phase I, first-in-human, open-label, proof-of-concept study with a single-arm, nonrandomized design aiming to assess safety and effects on glycemic after 6 months treatment of EDN procedure in T2DM patients. The study was performed at a single center in Nanjing, China (Center of Interventional Radiology and Vascular Surgery, Zhongda Hospital, Southeast University). The study protocol was approved by the Ethics Committee/Institutional Review Board for Clinical Research at our institution and complied with the recommendations of the Declaration of Helsinki. All patients signed written informed 
consent for the procedures performed and for their inclusion in this study. The study is registered under ClinicalTrials.gov (NCT 04086043).

\section{Patients}

Eligible patients were adults aged 18-75 years who were diagnosed with T2DM at least 5 years prior to enrollment. At the time of enrollment, the patients needed to have a serum $\mathrm{HbA1c}$ over $7.5 \%$, and have been on treatment of at least one oral antidiabetic agent (with or without insulin injection), with no changes in background anti-diabetic medication in the last 30 days. Patients were excluded if they had type 1 diabetes mellitus, arterial anatomy ineligible for EDN, aortic pathologies such as aneurysm or dissection that would preclude the EDN procedure, pregnant or intending to become pregnant within 1 year, orthostatic hypotension, eGFR $<30 \mathrm{ml} / \mathrm{min}$ (MDRD formula), acute or severe systemic infection, cerebral apoplexy or transient ischemic attack in the past 3 months, and acute coronary syndrome in the past 3 months.

\section{Study Procedure}

All procedures were performed by the same interventional radiologist (GJ.T., with more than 30 years of experience). A surface electrode was placed on the back of the patient and connected to the denervation device. Abdominal aortography was performed to identify the level of celiac artery (CA) and superior mesenteric artery (SMA) by means of transfemoral access with the use of an 8-F sheath and 5-F pigtail catheter. Then the EDN six-electrode catheter (Golden Leaf, Shanghai) was inserted to CA and aorta between CA and SMA through the sheath (Figure 1). The catheter was connected to the denervation device, and a test procedure was performed with the use of low-energy radiofrequencies to assess the adherence of the electrodes onto the blood vessel wall, judged by temperature and impedance changes: temperature $\sim 37^{\circ} \mathrm{C}$ and impedance $<400 \Omega$. The patients were under moderate analgesia with combinations of intravenous flurbiprofen and/or dezocine when EDN started. Denervation was carried out with ablation parameters set at time 120 seconds and temperature $60^{\circ} \mathrm{C}$. Impedance was also

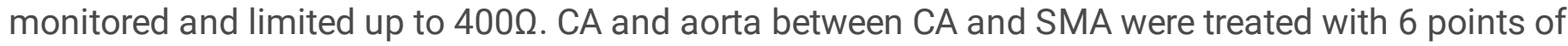
ablation, separately. After completion of the ablation, angiography of abdominal aorta was performed via the pigtail catheter. The puncture site was then closed with the use of Proglide vascular closure devices (Abbott Vascular, Abbott Park, Illinois).

\section{Outcome Measures and Assessments}

At baseline and follow-up visits, physical examination (including anthropometric measurements, systolic and diastolic BP) and laboratory assessment (HbA1c, fasting plasma glucose (FPG), 2-hour postprandial plasma glucose ( $2 \mathrm{hPG}$ ), fasting insulin, fasting C-peptide, plasma norepinephrine (NE), Angiotensin II (Ang II), liver biochemistry, and plasma lipids) were performed alongside recording of medication use and any adverse events (AEs). At each visit, the patients were asked for the occurrence of self-measured hypoglycemia (glucose level $<3.1 \mathrm{mmol} / \mathrm{L}$ )[24] and the occurrence of any other symptoms or AEs. AEs were graded according to the Common Terminology Criteria for Adverse Events (CTCAE v4.0) of the 
National Cancer Institute (Bethesda, Maryland). If there were any abnormalities found after the procedure, follow-up CT or CT angiography was performed.

Baseline measurements were used for further comparison. The anthropometrics, including waist circumference, body weight and body height, were measured as according to the WHO Guidelines. Measurements of office BP were done according to the ESH/ESC Guidelines[25] using an automatic BP Monitor (Omron Healthcare, Inc., Bannockburn, Illinois, USA). The food frequency questionnaire (FFQ25) [26] and international physical activity questionnaire (IPAQ)[27] were used as instrument for monitoring of diet and physical activity.

Comparisons were made between pre-procedure (baseline) and post-procedure metabolic parameters, lifestyle, and changes in background medication use. At baseline and during follow-up visits, C-peptide and insulin release tests were performed by a 75-g oral glucose tolerance test (OGTT) with plasma samples obtained at $0,30,60,120$, and 180 minutes after the glucose load. HOMA-IR was calculated with the use of the following equation: (FPG [in $\mathrm{mIU} / \mathrm{mL}$ ]) $\times$ (fasting insulin [in $\mathrm{mmol} / \mathrm{L}]$ )/22.5[28]. Post-EDN follow-up was planned for 2 years with visits scheduled at 3, 6, 12, 18 and 24 months. The primary efficacy endpoint was $\mathrm{HbA1c}$ at 6-month, and we report here data in 11 patients from the first 6 months of post-procedure follow-up.

\section{Statistical Analysis}

All statistical analyses were performed using SPSS statistics software (v18.0; IBM Corp, Somers, New York). Depending on the data distribution, continuous data were expressed as mean \pm standard deviation or median with interquartile range. Paired $t$ test or Wilcoxon signed-rank test were used to analyze the difference of parameters between baseline and 6-month follow-up. Significance of changes in FPG, 2hPG, systolic and diastolic BP at baseline and 1, 3, and 6 months of follow-ups were analyzed using one-way repeated measures ANOVA along with Bonferroni correction. All statistical tests were two sided at the a level of 0.05 unless stated otherwise.

\section{Results}

\section{Patient Characteristics}

Between September 2019 and July 2020, eleven patients were included. The baseline characteristics were listed in Table 1. The average age of all patients were $53.4 \pm 15.2$ years and 10 out of them were male. At baseline, only one patient was not using insulin injection, while the maximum daily insulin dose among them was $60 \mathrm{IU}$. All the eleven patients had finished 6-month follow-up.

\section{Safety}

Technical success was achieved in $100 \%$ of the treated patients. No severe treatment-related AEs or major complications, such as aneurysm or dissection (grade III or above according to CTCAE v4.0) were observed. Immediately after EDN, 4 patients (36.4\%) showed angiospasm on digital subtraction 
angiography, all of which resolved within 20 minutes. One patient (9.1\%) had nausea and vomiting, and two $(18.2 \%)$ had minor abdominal distension and constipation after EDN, which resolved within 3 days (Table 2).

\section{Changes of glycemic indices, medications, and lifestyle}

As shown in Table 3, the average HbA1c was reduced by $1.9 \%$ (9.9 \pm 1.6 compared to $8.0 \pm 2.4 \%$ at baseline, $P=0.005$ ) at 6 months of follow-up. The median HOMA-IR decreased from 13.3 (IQR 5.9-46.1) to 6.0 (IQR 3.1-11.9) $(P=0.016)$ (Figure 2A and B). At 1,3 , and 6 months of follow-ups, FPG levels were 10.2 \pm 0.8 , $10.1 \pm 1.2$, and $9.6 \pm 1.7 \mathrm{mmol} / \mathrm{L}$, respectively, compared to $15.4 \pm 1.6 \mathrm{mmol} / \mathrm{L}$ at baseline $(P=0.001$, < 0.001 , and $<0.001$, respectively), and $2 \mathrm{hPG}$ levels were $11.8 \pm 3.0,11.4 \pm 4.0$, and $11.0 \pm 5.9 \mathrm{mmol} / \mathrm{L}$, respectively, compared to $17.9 \pm 6.0 \mathrm{mmol} / \mathrm{L}$ at baseline $(P=0.001,0.001$, and 0.002 , respectively) (Figure 2C and D). There was no statistically significant change in fasting plasma insulin (185 (IQR 76.4-299.2) $\mathrm{nmol} / \mathrm{L}$ at baseline vs. 116.5 (IQR 79.1-217.7) $\mathrm{nmol} / \mathrm{L}$ at 6-mo follow-up $(P=0.075)$. In addition, OGTT results showed improvements in 3-hour C-peptide release test $\left(\mathrm{AUC}_{\mathrm{C} \text {-peptide }} 0.23\right.$ (IQR 0.18-0.32) pmol/mL vs. 0.28 (IQR 0.21-0.38) pmol/mL, $P=0.046$ ) (Figure 3).

The use of oral antidiabetic agents changed from 2 (IQR 2-2) to 2 (IQR 1-2) during follow-ups but without statistically significance $(P=0.059)$. In detail, 4 out of $11(36.4 \%)$ patients have quitted at least one kind of oral antidiabetic agents (one patient from 2 kinds to 1 , one from 2 to 0 , and two from 3 to 2 ). Since one of the patients was not on insulin injection at baseline, the change of insulin dose was measured in 10 patients. The results showed the median dose of insulin decreased from 24 (IQR 15.5-47) IU to 19 (IQR 927.5) IU at 6 months $(P=0.018)$, with the median dose reduction of 10 (IQR 0-20.25) IU. Two patients ceased insulin injection after EDN with one of them even coming off either insulin or oral antidiabetic agents.

Except that one patient had lumbar disc herniation one-month after EDN and stayed in bed for three months, diet and physical activity did not show significant change during follow-ups in the remaining 10 patients based on FFQ25 and IPAQ assessments.

\section{Changes of physical conditions, and other metabolic measurements}

At 6-month follow-up, weight, BMI and waist circumference did not show significant changes compared to those at baseline $(P=0.263, P=0.280, P=0.195$, respectively), as shown in Table 3 .

Systolic and diastolic BP showed no significant differences at baseline, 1-mo, 3-mo and 6-mo follow-ups ( $P=0.206, P=0.424$, respectively) (Figure 4A and B). Plasma NE level was 226.0 (IQR 186.0-258.4) $\mathrm{pg} / \mathrm{mL}$ at baseline and 205.8 (IQR 157.3-286.4) pg/mL at 6 months of follow-up ( $P=0.091$ ), and Ang II level was 74.3 (IQR 68.5-83.7) pg/mL at baseline and 73.0 (IQR 68.2-77.7) pg/mL at 6 months of followup $(P=0.091)$ (Figure 4C and D). 
As for plasma lipids, no significant differences were found in triglyceride (TG) (4.4 \pm 5.4 vs. $3.6 \pm 4.0$ $\mathrm{mmol} / \mathrm{L}, P=0.244)$, total cholesterol (TC) (5.1 \pm 1.8 vs. $4.6 \pm 1.1 \mathrm{mmol} / \mathrm{L}, P=0.157)$, high density

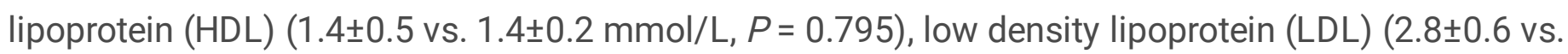
$2.4 \pm 0.6 \mathrm{mmol} / \mathrm{L}, P=0.080$ ) during follow-up (Figure 5). Additionally, improvements of liver biochemistry were observed during follow-ups. Alanine aminotransferase (ALT) levels decreased from 31.0 (IQR 25.046.0) U/L at baseline to 24.0 (IQR 14.0-38.0) U/L at 6-mo follow-up $(P=0.014)$, and $\gamma$-glutamyl transpeptidase (GGT) levels decreased from 47.0 (IQR 25.0-85.0) U/L at baseline to 27.0 (IQR 25.0-49.0) $\mathrm{U} / \mathrm{L}$ at 6-mo follow-up $(P=0.021)$, while there were no significant differences in aspertate aminotransferase (AST) and alkaline phosphatase (ALP) $(P=0.154, P=0.181$, respectively) (Figure 6).

\section{Discussion}

In this first-in-human study, a single-procedure EDN at new sites of CA and aorta between CA and SMA elicited a substantial improvement in glycemic control and improved insulin resistance in suboptimally controlled T2DM patients followed for 6 months, with an acceptable safety and tolerability profile observed to date.

The safety profile from this early clinical experience of EDN is encouraging. Patients who underwent the procedure experienced minimal intolerance. During the initial development of EDN, isolated cases of angiospasm were observed immediately after the procedure and resolved within 20 minutes without further sequelae. Few gastrointestinal symptoms were found after the procedure and resolved within 3 days, and no additional symptoms were reported in the follow-up period of 6 months, which is consist with our former research[23], indicating that EDN is a feasible and safe technique.

Catheter-based denervation has been used in clinic since the past decade[10], and researchers were still working on different targets, such as common hepatic artery[20,29] and celiac artery[30], where reduced sympathetic activity and decreased BP were found as well. In this study, EDN was conducted at new sites of CA and aorta between CA and SMA in T2DM patients to evaluate the effects on glycemic control. Improvements of FPG and 2hPG were observed 6 months post EDN. Mean HbA1c was 1.9\% lower at 6 months post EDN compared with baseline, accompanied with a median HOMA-IR decrease from 13.3 to 6.0. These results suggested a significant improvement in glycemic control possibly driven by the remission of insulin resistance, which was hardly seen in RDN treated patients[31]. Therefore, we hypothesized that different from the indirect control of RDN on hepatic glucose metabolism, EDN had a straightforward influence on it through directly turning down the sympathetic output to the liver[32]. This hypothesis is consistent with an animal-based study considering denervation in common hepatic arteries lessened glucose intolerance[20]. According to C-peptide and insulin release tests, EDN could lead to improvements in beta-cell function, which was previously observed in RDN-treated patients[33]. Based on the fact that sympathetic nerve innervates the islet through the celiac ganglia and thus suppress the secretion of insulin[14], the possible explanation could be that EDN, to some extent, directly or indirectly reduced the sympathetic output into islet. 
In this study, medication remained unchanged post EDN unless plasma glucose was well controlled. Several patients reduced their oral antidiabetic agents and dose of insulin injection through 6 months of follow-up, and two patients stopped using insulin after EDN with one of them came off either oral antidiabetic agents or insulin injection. A consistent and durable decrease of FPG and 2hPG levels was also observed in most of the patients, except one patient had lumbar disc herniation one-month after EDN and stayed in bed for three months, whose FPG and 2hPG levels reduced at 1-mo follow-up but increased at 3-mo and 6-mo follow-ups. According to FFQ25 and IPAQ assessments, diet and physical activity did not change during follow-ups in the remaining 10 patients, so the early improvement in glycemic control induced by EDN is unlikely to be explained by decreased caloric intake or increased physical activity.

Office BP was also measured in this study, and both systolic and diastolic BP showed no statistically significant changes after EDN. Since RDN could decrease BP through reducing sympathetic activity[34], serum NE and Ang II levels[35] were tested in this study to investigate whether EDN could have influence on sympathetic activity. Although NE and Ang II levels were within the normal range in all the eleven patients before and after the procedure and changes were not statistically significant, they decreased in value at 6-mo follow-up when compared with baseline, suggesting that EDN could somehow lower sympathetic activity.

A durable decrease in ALT and GGT was also observed 6-mo after EDN. It is well recognized that insulin resistance is thought to be a common pathological driver in patients with T2DM and non-alcoholic fatty liver disease (NAFLD) [36, 37]. The reduction of ALT and GGT observed with EDN suggests an additional beneficial effect of EDN on concomitant NAFLD measures, which is consistent with a recently published animal study showing that manipulation of the liver sympathetic nerves may represent a novel therapeutic strategy for NAFLD[29]. Most of ALT and GGT levels and the pre-procedure ALT and GGT measurement were normal (31.0 U/L for ALT, and 47.0 U/L for GGT), so the clinical relevance of a decrease to more normal level can be questioned. A liver Magnetic Resonance Proton Density Fat Fraction would have added value here. Therefore, effects of EDN on the liver need to be examined in the future. Furthermore, a trend of lipidemia improvement, although not statistically significant, was observed in this cohort. This was consistent with the findings in a basic research[38]. The remission of insulin resistance and the improvement of liver lipid metabolism may potentially contribute to this phenomenon[29, 39].

Since insulin sensitivity improves after EDN, we speculated that patients with greater beta-cell reserve (high C-peptide and insulin baseline levels)[24] might benefit more from this procedure than patients with lower beta-cell reserve. However, we did not find a clear association between baseline C-peptide or insulin levels and later glycemic outcome, which is probably due to the inter-individual differences, relatively small sample size and lack of power to demonstrate a difference in this parameter. Nevertheless, the precise mechanism underlying the effects of EDN is not well understood: changes in sympathetic activity[30], gut microbiota[40, 41], lipid metabolism[31] or liver glycogen metabolism[20] are possible mechanisms of action. Further mechanistic studies to unravel the potential mechanisms underlying the effect of EDN on the liver, beta-cell function, adipose tissue, inflammation[42], and sympathetic activity 
are eagerly awaited. Meanwhile, whether EDN will affect gastrointestinal motility, especially gastric emptying is worth studying as well, and this would throw light on the potential benefits or complications of EDN.

Any first-in-human study comes with shortcomings, and the small sample size along with the lack of an appropriate control would have impeded us from better measuring the effect of EDN and the pattern of change over time. Meanwhile, HOMA-IR was used to assess the insulin sensitivity, whereas the "criterion standard" is the hyperinsulinemic-euglycemic step clamp and there was no available equipment in our hospital at that time. Additional limits include lack of liver imaging, histological data, advanced measurement of sympathetic activity (heart rate variability or muscle sympathetic nerve activity), and other hepatic confounders. These factors will be further addressed in future studies.

\section{Conclusions}

This study suggested that EDN at new sites of CA and aorta between CA and SMA using the novel sixelectrode catheter system elicits a clinically significant improvement in glycemic control and moderate improvement of insulin resistance in patients with T2DM, with good tolerability. Achievement of such metabolic benefit through a minimally interventional treatment offers a potentially new therapeutic solution for patients with T2DM. The EDN approach may overcome treatment adherence and compliance issues, a major shortcoming of all pharmacological approaches. Further work is necessary to better understand the clinical utility of this procedure-based intervention in controlled trial conditions in larger numbers of patients, and key questions will focus on establishing efficacy and the magnitude and durability of effect and understanding the accurate mechanisms.

\section{Declarations}

Ethics approval and consent to participate: The study protocol was approved by the Ethics Committee/Institutional Review Board for Clinical Research at our institution and complied with the recommendations of the Declaration of Helsinki. All patients signed written informed consent for the procedures performed and for their inclusion in this study.

Consent for publication: Not applicable.

Availability of data and materials: The datasets used and/or analyzed during the current study are available from the corresponding author on reasonable request.

Competing interests: The authors declare that they have no competing interests.

Funding: This work is supported by the National Major Program of Research \& Development of China (2018YFA0704100), the Major Program of Scientific Instrument of the National Natural Science Foundation of China (81827805), and Clinical Medicine Center of Jiangsu Province (Innovation Section) (YXZXA2016005). 
Authors' contributions: GJT conceived the study and designed the main concept of the study. TP, QW, ZW, $Y Q$ and $R L$ analyzed the data. TP and ZW wrote the first version of the manuscript and edited the manuscript. TP, QW, QZ, DCL, and YW acquired the data and interpreted the results. GJT, LL and ZLS critically revised the important intellectual content of the manuscript. All authors contributed to the discussion, reviewed the manuscript, and approved the final draft submitted.

Acknowledgements: Not applicable.

Authors' information: ${ }^{1}$ Center of Interventional Radiology and Vascular Surgery, Department of Radiology, Zhongda Hospital, Medical School, Southeast University, 87 Dingjiaqiao Road, Nanjing 210009, China; ${ }^{2}$ Department of Endocrinology, ZhongDa Hospital, School of Medicine, Southeast University; Institute of Pancreas, Southeast University, 87 Dingjiaqiao Road, Nanjing 210009, China; ${ }^{3}$ Department of Anesthesiology, Nanjing Drum Tower Hospital, Medical School of Nanjing University, 321 Zhongshan Road, Nanjing 210008, China; ${ }^{4}$ Department of Endocrinology, ZhongDa Hospital, School of Medicine, Southeast University; Department of Clinical Science and Research, ZhongDa Hospital, School of Medicine, Southeast University, 87 Dingjiaqiao Road, Nanjing 210009, China; ${ }^{5}$ Department of Endocrinology, ZhongDa Hospital, School of Medicine, Southeast University, 87 Dingjiaqiao Road, Nanjing 210009, China

\section{Abbreviations}

T2DM: type 2 diabetes mellitus; SNS: sympathetic nervous system; RDN: renal denervation; BP: blood pressure; HbA1c: glycosylated hemoglobin; EDN: endovascular denervation; CA: celiac artery; SMA: superior mesenteric artery; FPG: plasma glucose; 2hPG: 2-hour postprandial plasma glucose; NE: norepinephrine; Ang II: Angiotensin II; AEs: adverse events; FFQ25: food frequency questionnaire; IPAQ: international physical activity questionnaire; OGTT: oral glucose tolerance test; BMI: body mass index; TG: triglyceride; TC: total cholesterol; HDL: high density lipoprotein; LDL: Iow density lipoprotein; ALT: alanine aminotransferase; GGT: $\gamma$-glutamyl transpeptidase; AST: aspertate aminotransferase; ALP: alkaline phosphatase; AUC: area under curve; NAFLD: non-alcoholic fatty liver disease.

\section{References}

1. Guariguata L, Whiting DR, Hambleton I, Beagley J, Linnenkamp U, Shaw JE. Global estimates of diabetes prevalence for 2013 and projections for 2035. Diabetes Res Clin Pract. 2014; 103: 137-149.

2. Facilitating Behavior Change and Well-being to Improve Health Outcomes: Standards of Medical Care in Diabetes-2021. Diabetes Care. 2021; 44(Suppl 1): S53-S72.

3. Obesity Management for the Treatment of Type 2 Diabetes: Standards of Medical Care in Diabetes2021. Diabetes Care. 2021; 44(Suppl 1): S100-S110.

4. Pharmacologic Approaches to Glycemic Treatment: Standards of Medical Care in Diabetes-2021. Diabetes Care. 2021; 44(Suppl 1): S111-S124. 
5. Egede LE, Gebregziabher M, Dismuke CE, Lynch CP, Axon RN, Zhao Y, et al. Medication nonadherence in diabetes: longitudinal effects on costs and potential cost savings from improvement. Diabetes Care. 2012; 35: 2533-2539.

6. Davies MJ, D'Alessio DA, Fradkin J, Kernan WN, Mathieu C, Mingrone G, et al. Management of hyperglycaemia in type 2 diabetes, 2018. A consensus report by the American Diabetes Association (ADA) and the European Association for the Study of Diabetes (EASD). Diabetologia. 2018; 61: 24612498.

7. Masuo K, Mikami H, Ogihara T, Tuck ML. Sympathetic nerve hyperactivity precedes hyperinsulinemia and blood pressure elevation in a young, nonobese Japanese population. Am J Hypertens. 1997; 10: 77-83.

8. Huggett RJ, Scott EM, Gilbey SG, Stoker JB, Mackintosh AF, Mary DA. Impact of type 2 diabetes mellitus on sympathetic neural mechanisms in hypertension. Circulation. 2003; 108: 3097-3101.

9. Grassi G, Dell'Oro R, Quarti-Trevano F, Scopelliti F, Seravalle G, Paleari F, et al. Neuroadrenergic and reflex abnormalities in patients with metabolic syndrome. Diabetologia. 2005; 48: 1359-1365.

10. Krum H, Schlaich M, Whitbourn R, Sobotka PA, Sadowski J, Bartus K, et al. Catheter-based renal sympathetic denervation for resistant hypertension: a multicentre safety and proof-of-principle cohort study. Lancet. 2009; 373: 1275-1281.

11. Mahfoud F, Schlaich M, Kindermann I, Ukena C, Cremers B, Brandt MC, et al. Effect of renal sympathetic denervation on glucose metabolism in patients with resistant hypertension: a pilot study. Circulation. 2011; 123: 1940-1946.

12. Witkowski A, Prejbisz A, Florczak E, Kądziela J, Śliwiński P, Bieleń P, et al. Effects of renal sympathetic denervation on blood pressure, sleep apnea course, and glycemic control in patients with resistant hypertension and sleep apnea. Hypertension. 2011; 58: 559-565.

13. Verloop WL, Spiering W, Vink EE, Beeftink MM, Blankestijn PJ, Doevendans PA, et al. Denervation of the renal arteries in metabolic syndrome: the DREAMS-study. Hypertension. 2015; 65: 751-757.

14. Ahrén B. Autonomic regulation of islet hormone secretion-implications for health and disease. Diabetologia. 2000; 43: 393-410.

15. McCuskey RS. Anatomy of efferent hepatic nerves. Anat Rec A Discov Mol Cell Evol Biol. 2004; 280: 821-826.

16. Lin EE, Scott-Solomon E, Kuruvilla R. Peripheral Innervation in the Regulation of Glucose Homeostasis. Trends Neurosci. 2021; 44: 189-202.

17. Rosas-Ballina M, Ochani M, Parrish WR, Ochani K, Harris YT, Huston JM, et al. Splenic nerve is required for cholinergic antiinflammatory pathway control of TNF in endotoxemia. Proc Natl Acad Sci U S A. 2008; 105: 11008-11013.

18. Bartness TJ, Liu Y, Shrestha YB, Ryu V. Neural innervation of white adipose tissue and the control of lipolysis. Front Neuroendocrinol. 2014; 35: 473-493.

19. Blaszkiewicz M, Willows JW, Johnson CP, Townsend KL. The Importance of Peripheral Nerves in Adipose Tissue for the Regulation of Energy Balance. Biology (Basel). 2019 ;8:10. 
20. Kraft G, Vrba A, Scott M, Allen E, Edgerton DS, Williams PE, et al. Sympathetic Denervation of the Common Hepatic Artery Lessens Glucose Intolerance in the Fat- and Fructose-Fed Dog. Diabetes. 2019; 68: 1143-1155.

21. Kandzari DE, Böhm M, Mahfoud F, Townsend RR, Weber MA, Pocock S, et al. Effect of renal denervation on blood pressure in the presence of antihypertensive drugs: 6-month efficacy and safety results from the SPYRAL HTN-ON MED proof-of-concept randomised trial. Lancet. 2018; 391: 2346-2355.

22. Townsend RR, Mahfoud F, Kandzari DE, Kario K, Pocock S, Weber MA, et al. Catheter-based renal denervation in patients with uncontrolled hypertension in the absence of antihypertensive medications (SPYRAL HTN-OFF MED): a randomised, sham-controlled, proof-of-concept trial. Lancet. 2017; 390: 2160-2170.

23. Zhang Q, Guo JH, Zhu HD, Zhong YM, Pan T, Yin HQ, et al. Endovascular Denervation: A New Approach for Cancer Pain Relief? J Vasc Interv Radiol. 2018; 29: 1639-1644.

24. van Baar ACG, Holleman F, Crenier L, Haidry R, Magee C, Hopkins D, et al. Endoscopic duodenal mucosal resurfacing for the treatment of type 2 diabetes mellitus: one year results from the first international, open-label, prospective, multicentre study. Gut. 2020; 69: 295-303.

25. Mancia G, Fagard R, Narkiewicz K, Redon J, Zanchetti A, Böhm M, et al. 2013 ESH/ESC guidelines for the management of arterial hypertension: the Task Force for the Management of Arterial Hypertension of the European Society of Hypertension (ESH) and of the European Society of Cardiology (ESC). Eur Heart J. 2013; 34: 2159-2219.

26. Mikkelsen TB, Osler M, Olsen SF. Validity of protein, retinol, folic acid and n-3 fatty acid intakes estimated from the food-frequency questionnaire used in the Danish National Birth Cohort. Public Health Nutr. 2006; 9: 771-778.

27. Romain C, Chung LH, Marín-Cascales E, Rubio-Arias JA, Gaillet S, Laurent C, et al. Sixteen Weeks of Supplementation with a Nutritional Quantity of a Diversity of Polyphenols from Foodstuff Extracts Improves the Health-Related Quality of Life of Overweight and Obese Volunteers: A Randomized, Double-Blind, Parallel Clinical Trial. Nutrients. 2021;13:492.

28. Pan T, Guo JH, Ling L, Qian Y, Dong YH, Yin HQ, et al. Effects of Multi-Electrode Renal Denervation on Insulin Sensitivity and Glucose Metabolism in a Canine Model of Type 2 Diabetes Mellitus. J Vasc Interv Radiol. 2018; 29: 731-738.

29. Hurr C, Simonyan H, Morgan DA, Rahmouni K, Young CN. Liver sympathetic denervation reverses obesity-induced hepatic steatosis. J Physiol. 2019; 597: 4565-4580.

30. Asirvatham-Jeyaraj N, Gauthier MM, Banek CT, Ramesh A, Garver H, Fink GD, et al. Renal Denervation and Celiac Ganglionectomy Decrease Mean Arterial Pressure Similarly in Genetically Hypertensive Schlager (BPH/2J) Mice. Hypertension. 2021; 77: 519-528.

31. Zhang Z, Liu K, Xiao S, Chen X. Effects of catheter-based renal denervation on glycemic control and lipid levels: a systematic review and meta-analysis. Acta Diabetol. 2021; [Epub ahead of print]. 
32. Iyer MS, Bergman RN, Korman JE, Woolcott OO, Kabir M, Victor RG, et al. Renal Denervation Reverses Hepatic Insulin Resistance Induced by High-Fat Diet. Diabetes. 2016; 65: 3453-3463.

33. Ott C, Schmid A, Mahfoud F, Akarca E, Kistner I, Ditting T, et al. Secretory Capacity of Pancreatic BetaCells Is Enhanced 6 Months After Renal Denervation in Hypertensive Patients. J Am Coll Cardiol. 2018; 72: 3372-3374.

34. Ukena C, Seidel T, Rizas K, Scarsi D, Millenaar D, Ewen S, et al. Effects of renal denervation on 24-h heart rate and heart rate variability in resistant hypertension. Clin Res Cardiol. 2020; 109: 581-588.

35. Sharp TE, 3rd, Polhemus DJ, Li Z, Spaletra P, Jenkins JS, Reilly JP, et al. Renal Denervation Prevents Heart Failure Progression Via Inhibition of the Renin-Angiotensin System. J Am Coll Cardiol. 2018; 72: 2609-2621.

36. Guerra S, Gastaldelli A. The role of the liver in the modulation of glucose and insulin in non alcoholic fatty liver disease and type 2 diabetes. Curr Opin Pharmacol. 2020; 55: 165-174.

37. Adams LA, Lymp JF, St Sauver J, Sanderson SO, Lindor KD, Feldstein A, et al. The natural history of nonalcoholic fatty liver disease: a population-based cohort study. Gastroenterology. 2005; 129: 113121.

38. Bruinstroop E, Eliveld J, Foppen E, Busker S, Ackermans MT, Fliers E, et al. Hepatic denervation and dyslipidemia in obese Zucker (fa/fa) rats. Int J Obes (Lond) (2005). 2015; 39: 1655-1658.

39. Rodriguez-Colon SM, Mo J, Duan Y, Liu J, Caulfield JE, Jin X, et al. Metabolic syndrome clusters and the risk of incident stroke: the atherosclerosis risk in communities (ARIC) study. Stroke. 2009; 40: 200-205.

40. Khan MT, Nieuwdorp M, Bäckhed F. Microbial modulation of insulin sensitivity. Cell Metab. 2014; 20 : 753-760.

41. Zhang Q, Hu N. Effects of Metformin on the Gut Microbiota in Obesity and Type 2 Diabetes Mellitus. Diabetes Metab Syndr Obes. 2020; 13: 5003-5014.

42. Xiao L, Kirabo A, Wu J, Saleh MA, Zhu L, Wang F, et al. Renal Denervation Prevents Immune Cell Activation and Renal Inflammation in Angiotensin II-Induced Hypertension. Circ Res. 2015; 117: 547557.

\section{Tables}

\section{Table 1 Baseline demographic and clinical characteristics of the intention-to-treat population}




\begin{tabular}{lc}
\hline Patient characteristics & Value $(\mathrm{n}=11)$ \\
\hline Age, years (range) & $53.4 \pm 15.2(28-72)$ \\
Sex, n (\%) & $1(9.1)$ \\
$\quad$ Female & $10(90.9)$ \\
$\quad$ Male & $73.2 \pm 15.3(51-104)$ \\
Weight (kg) & $25.8 \pm 6.0(19.7-40.6)$ \\
BMI (kg/m ${ }^{2}$ ) & $91.3 \pm 11.5(78-114)$ \\
Waist circumference (cm) & $142.8 \pm 17.7(122-176)$ \\
Systolic blood pressure (mmHg) & $86.0 \pm 12.4(70-110)$ \\
Diastolic blood pressure (mmHg) & $15.1 \pm 7.3(5-28)$ \\
Duration of type 2 diabetes, years (range) & $9.9 \pm 1.6(7.5-11.4)$ \\
HbA1c (\%) & $15.4 \pm 5.4(7.7-27.0)$ \\
FPG (mmol/L) & $2.0 \pm 0.6(1-3)$ \\
Oral antidiabetic agents (kinds) & $27.4 \pm 18.3(0-60)$ \\
Insulin injection (IU) &
\end{tabular}

Data are mean \pm SD or $n(\%)$, unless otherwise indicated.

HbA1c = glycosylated hemoglobin; FPG = fasting plasma glucose .

Table 2 Treatment-related adverse events

\begin{tabular}{lc}
\hline Adverse events & $\begin{array}{c}\text { No (\%) of patients } \\
(\mathrm{n}=11)\end{array}$ \\
\hline Angiospasm & $4(36.4)$ \\
Nausea and vomiting & $1(9.1 \%)$ \\
Minor abdominal distension and constipation & $2(18.2 \%)$ \\
Self-measured hypoglycemia & 0 \\
Orthostatic hypotension & 0 \\
Aneurysm & 0 \\
Dissection & 0 \\
\hline
\end{tabular}


Table 3 Changes in anthropometric, medication and metabolic measurements 


\section{Glycemic indices}

HbA1c (\%)

$9.9 \pm 1.6$

$8.0 \pm 2.4$

$0.005^{*}$

HOMA-IR

$13.3(5.9-46.1)$

$6.0(3.1-11.9)$

$0.016^{*}$

FPG (mmol/L)

$$
15.4 \pm 5.4
$$

$10.1 \pm 4.0$

$<$

$0.001^{\dagger}$

2hPG (mmol/L)

$17.9 \pm 6.0$

185 (76.4-

299.2)

$0.23(0.18-0.32)$

$0.47(0.23-1.26)$

$11.4 \pm 4.0$

$0.001^{\dagger}$

Fasting plasma insulin (nmol/L)

$\mathrm{AUC}_{\mathrm{C} \text {-peptide }}(\mathrm{pmol} / \mathrm{mL})$

$\mathrm{AUC}_{\text {Insulin }}(\mathrm{pmol} / \mathrm{mL})$

Antidiabetic medication

Oral antidiabetic agents (kinds)

Daily insulin injection (IU) $(n=10)$

$2.0(2.0-2.0)$

$2.0(1.0-2.0)$

0.059

24 (15.5-47)

19 (9-27.5)

$0.018^{*}$

Physical conditions

Weight (kg)

$73.2 \pm 15.3$

$70.5 \pm 9.1$

0.263

BMI $\left(\mathrm{kg} / \mathrm{m}^{2}\right)$

$25.8 \pm 6.0$

$24.8 \pm 3.3$

0.280

Waist circumference (cm)

$91.3 \pm 11.5$

$89.5 \pm 9.2$

0.195

BP (mmHg)

Systolic BP

$\begin{array}{lll}142.8 \pm 17.7 & 136.9 \pm 8.9 & 0.298^{\dagger} \\ 86.0 \pm 12.4 & 82.5 \pm 7.5 & 0.300^{\dagger}\end{array}$

Diastolic BP

Sympathetic nervous system related hormones (pg/mL)

NE

$226.0(186.0$

258.4)

Ang II

74.3 (68.5-83.7)

205.8 (157.3-

0.091

286.4)

$74.3(68.5-83.7)$

$73.0(68.2-77.7)$

0.091 
Liver biochemistry (U/L)

ALT

AST

ALP

GGT

Plasma lipids (mmol/L)

TG

TC

HDL

LDL
31.0 (25.0-46.0) $24.0(14.0-38.0) \quad 0.014^{*}$

$24.0(19.0-40.0) \quad 21.0(16.0-33.0) \quad 0.154$

$84.0(64.0-\quad 68.0(65.0-91.0) \quad 0.181$

104.0)

$47.0(25.0-85.0) \quad 27.0(25.0-49.0) \quad 0.021^{*}$

Data are mean \pm SD or median with IQR

* $P<0.05$

$\dagger P<0.05 / 6=0.008$ according to Bonferroni correction

HbA1c: glycosylated hemoglobin; HOMA-IR: homeostasis-model assessment of insulin resistance; FPG: fasting plasma glucose; 2hPG: 2-hour postprandial plasma glucose; AUC: area under curve; BMI: body mass index; BP; blood pressure; NE: norepinephrine; Ang II: angiotensin II; TG: triglyceride; TC: total cholesterol; HDL: high density lipoprotein; LDL: low density lipoprotein; ALT: alanine aminotransferase; AST: aspertate aminotransferase; ALP: alkaline phosphatase; GGT: $\gamma$-glutamyl transpeptidase.

\section{Figures}




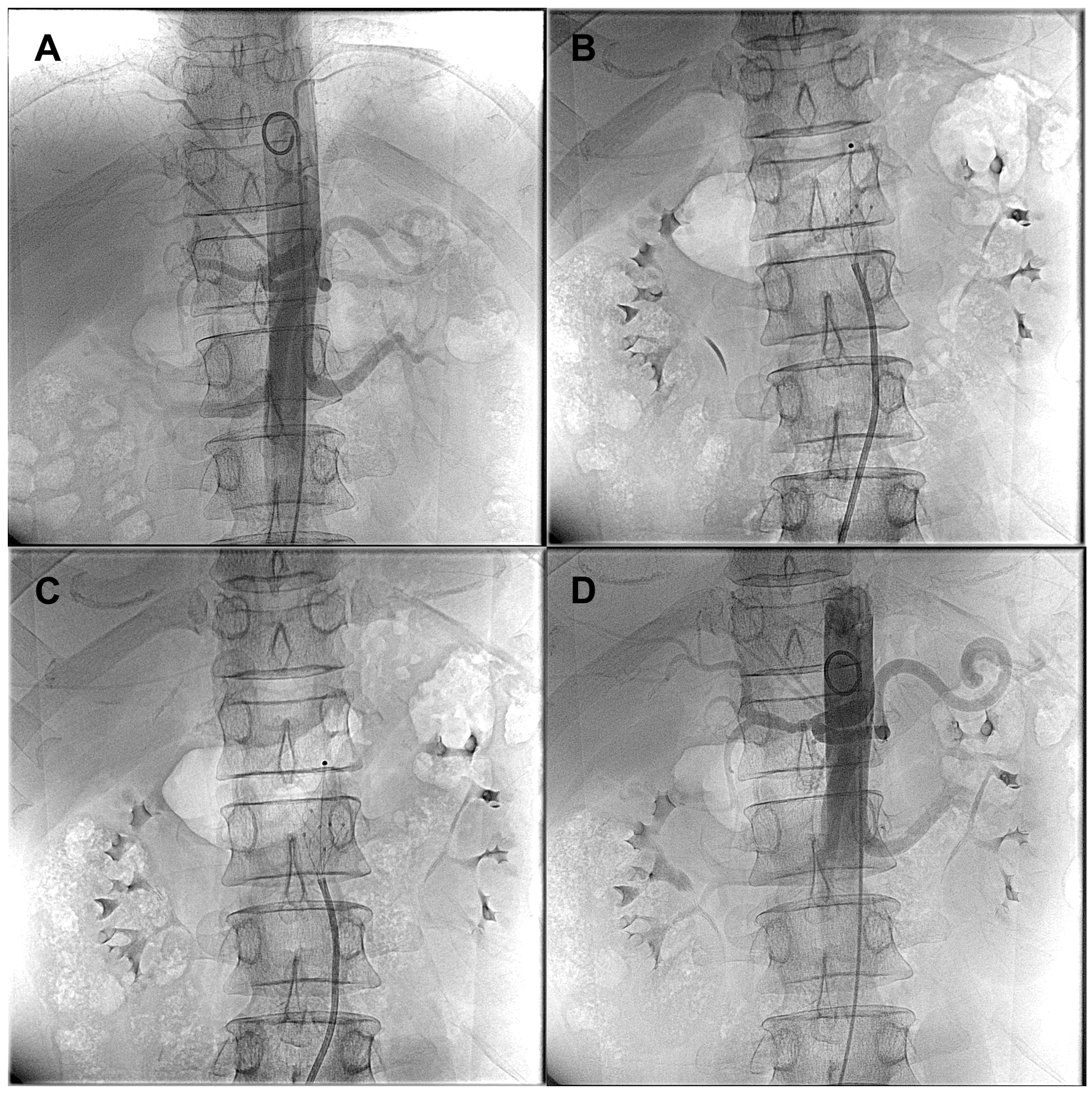

\section{Figure 1}

Images during endovascular denervation. (A) Digital subtraction angiography of the abdominal aorta before endovascular denervation. (B) The 6-electrode catheter was inserted to the celiac artery. (C) The 6electrode catheter was inserted to aorta between celiac artery and the superior mesenteric artery. (D) Digital subtraction angiography of the abdominal aorta immediately after endovascular denervation. 
A

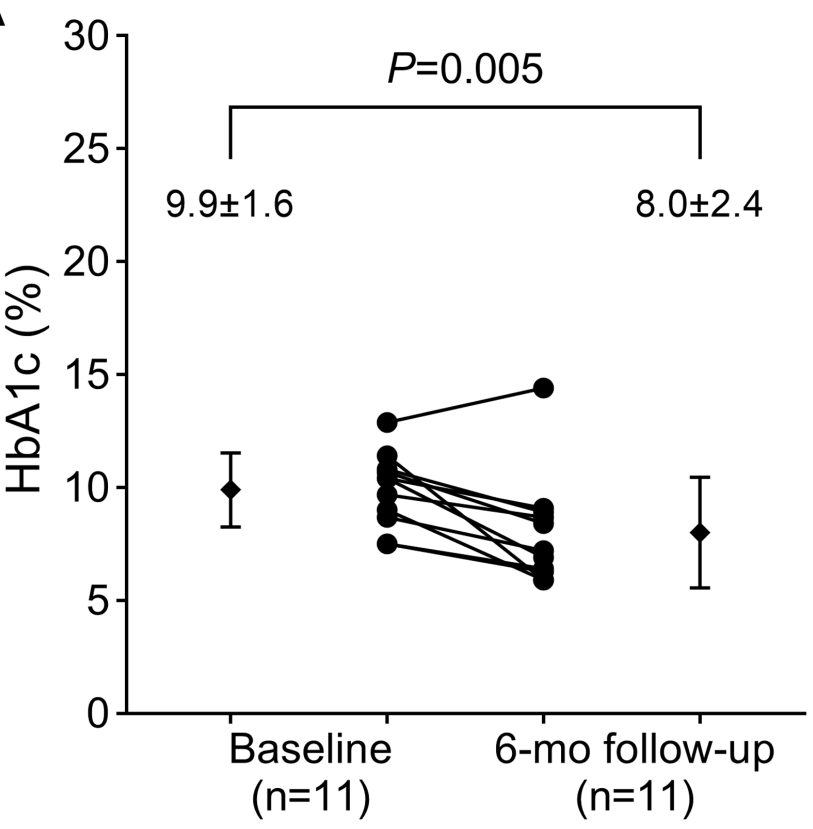

C

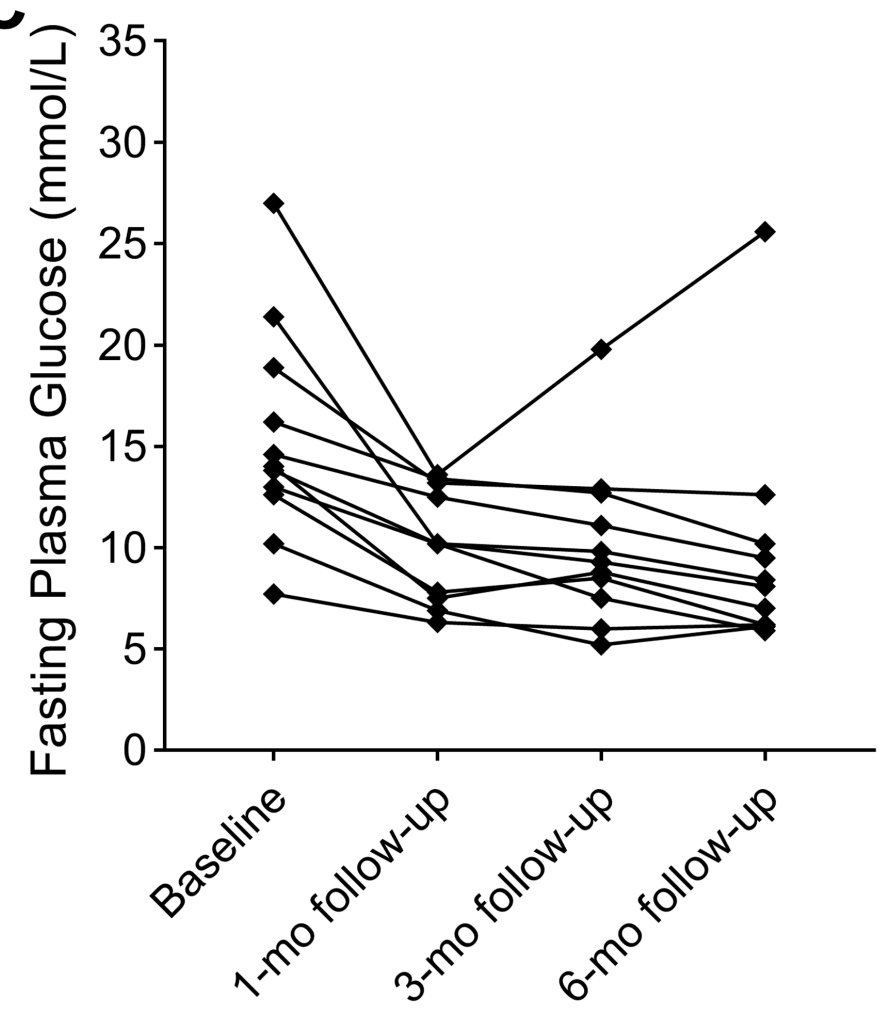

B

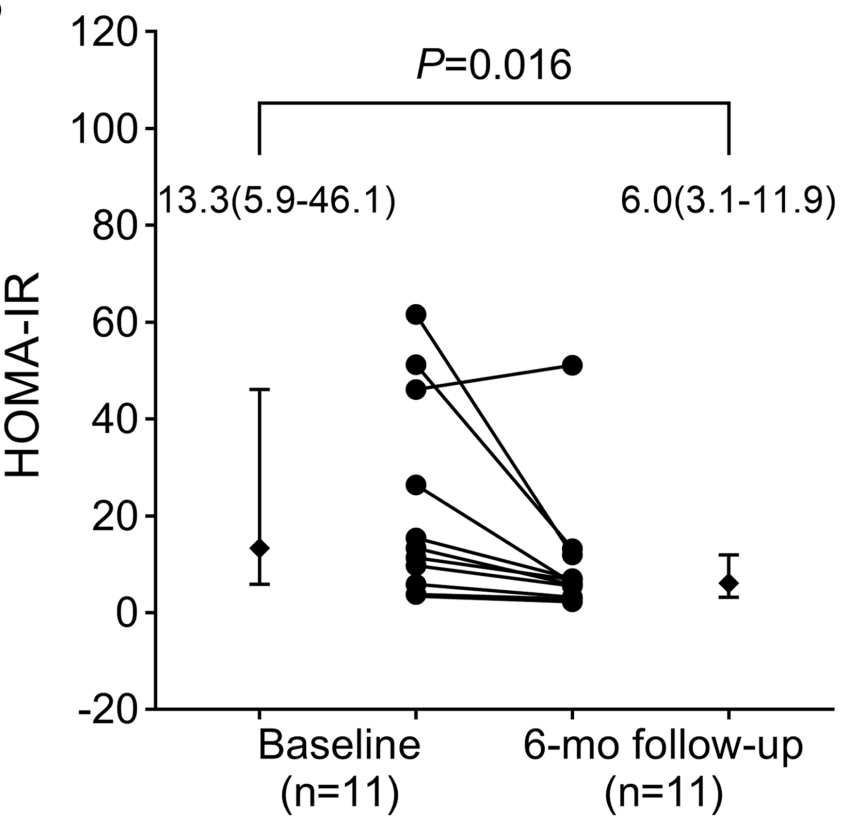

D

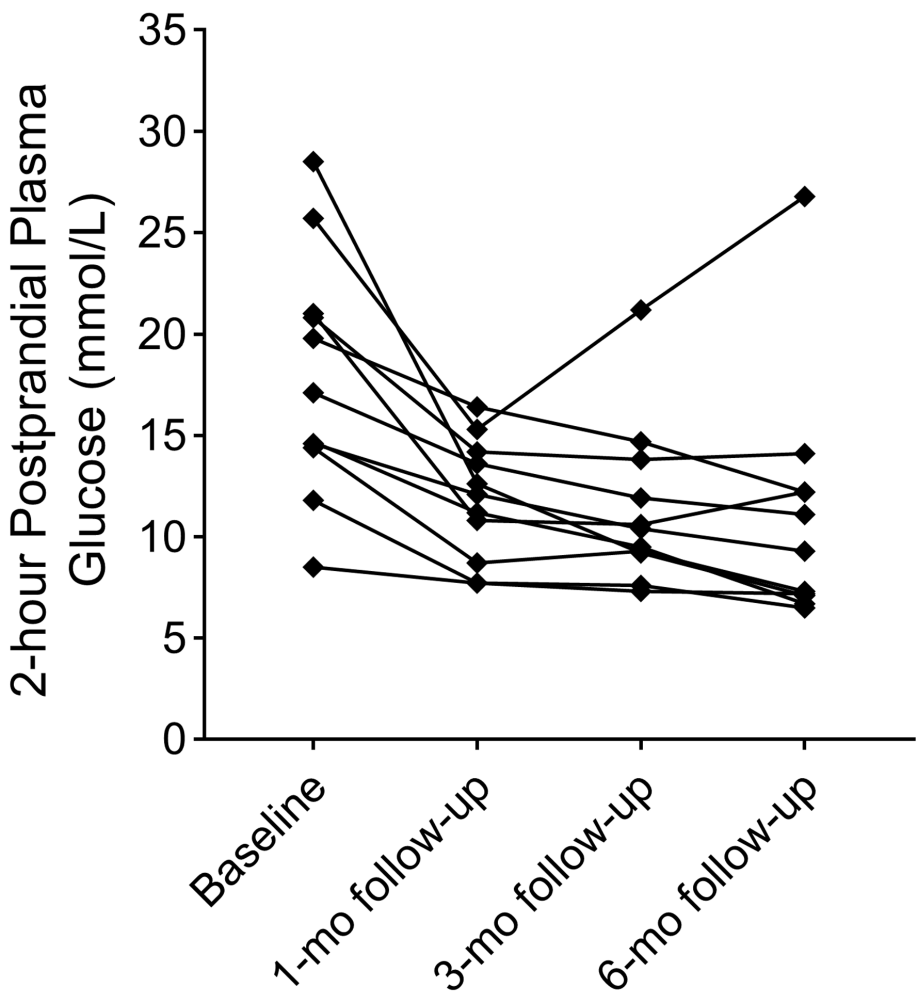

Figure 2

Changes of glycemic indices between baseline and 6-month follow-up. (A) Glycosylated hemoglobin. (B) Homeostasis-model assessment of insulin resistance. (C) Fasting plasma glucose. (D) 2-hour postprandial plasma glucose. 

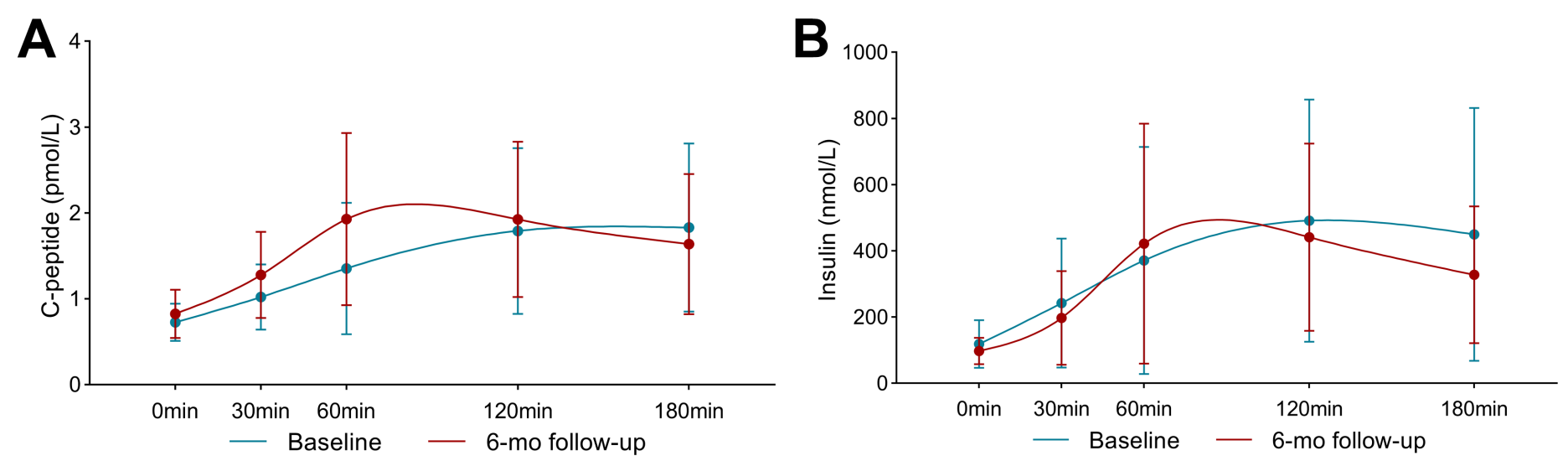

Figure 3

Changes in C-peptide and insulin release tests between baseline and 6-month follow-up. (A) C-peptide release tests. (B) Insulin release tests. 
A

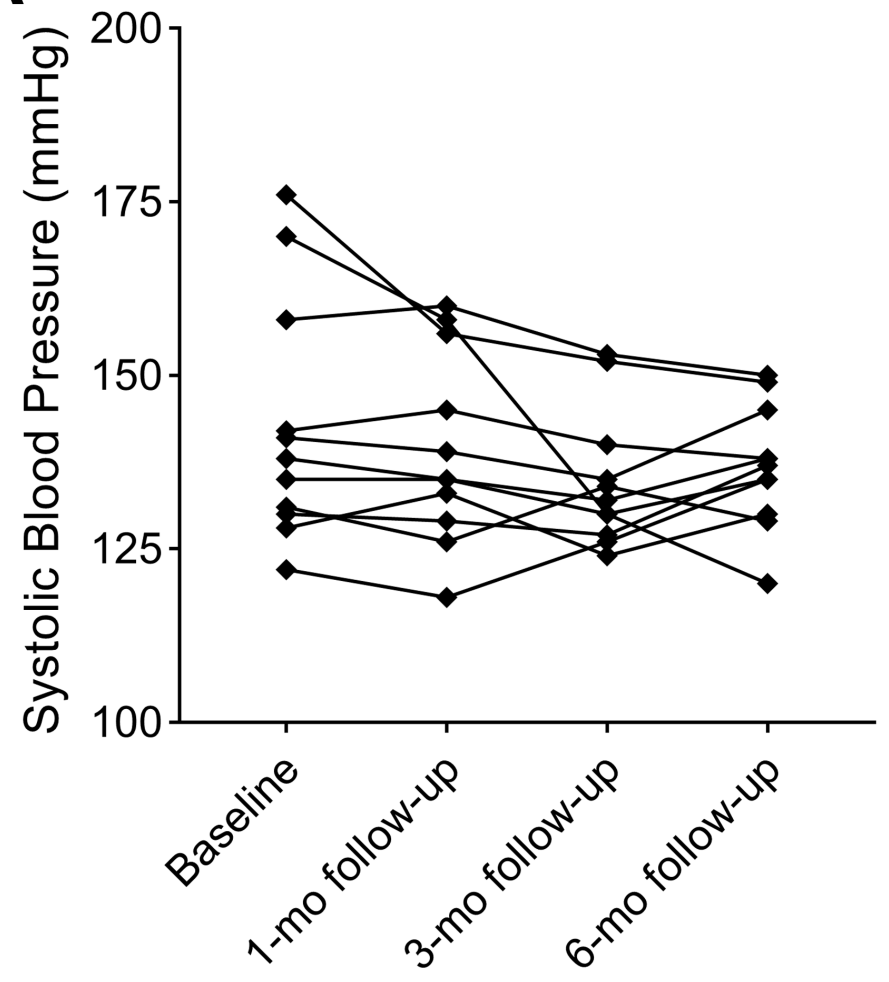

C

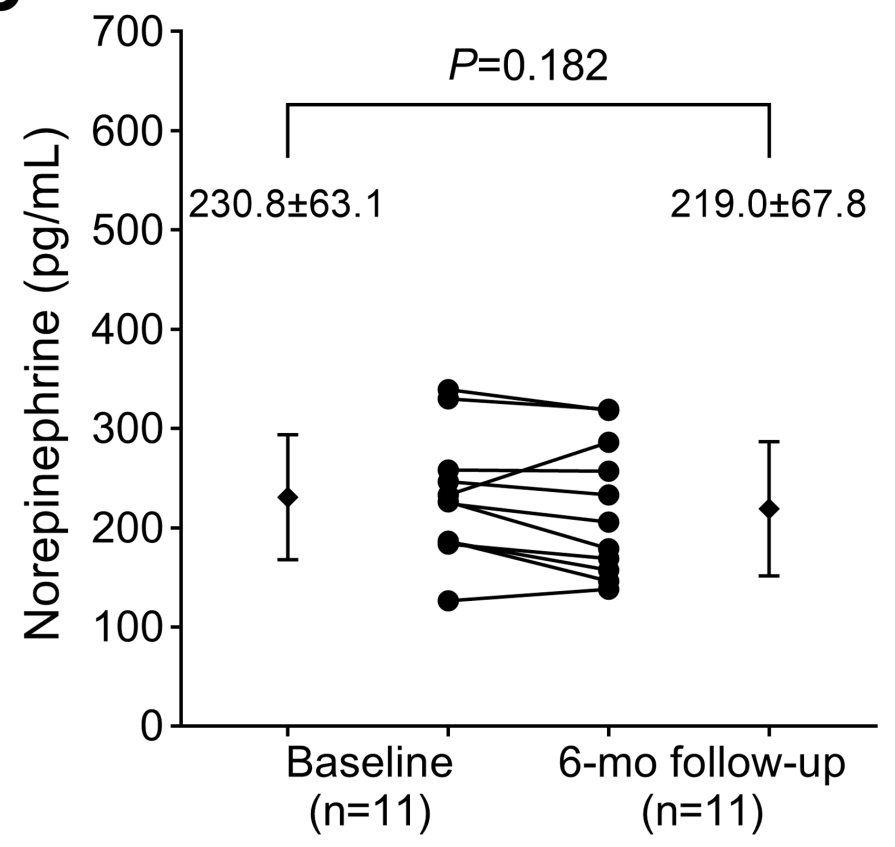

B

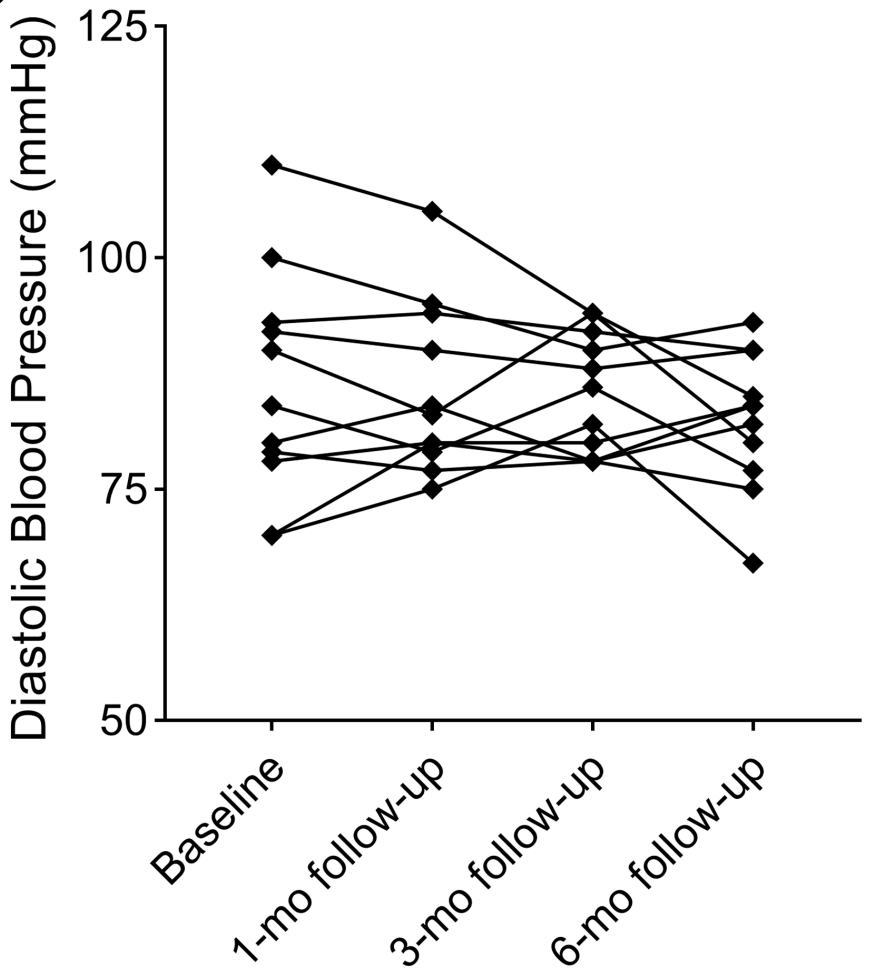

D

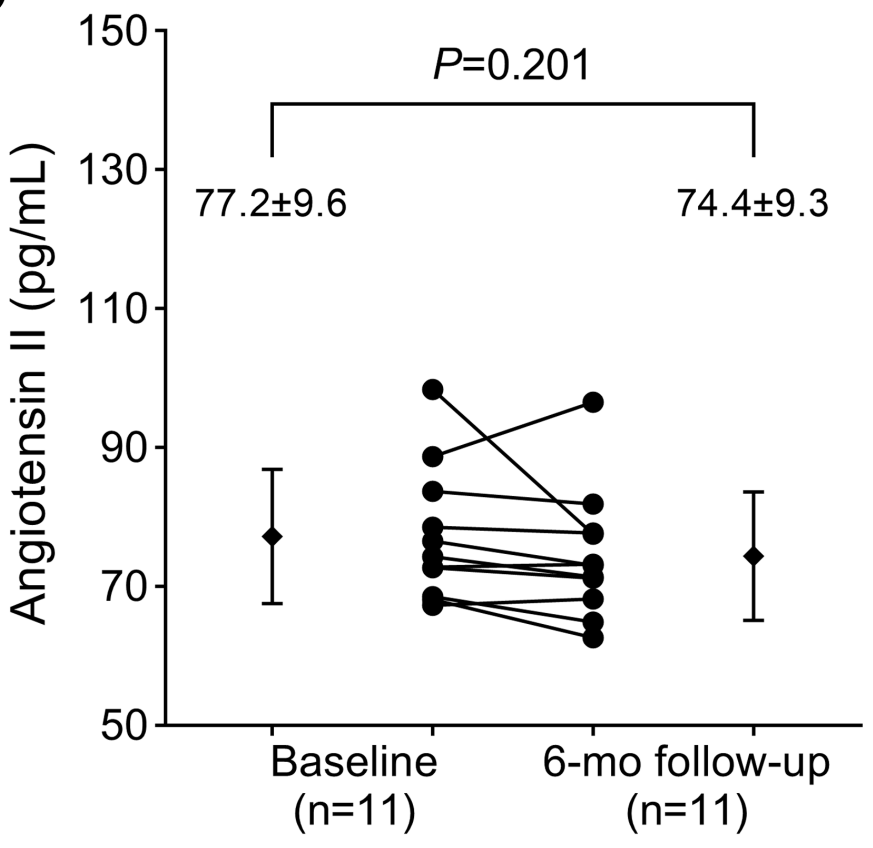

Figure 4

Changes of blood pressure, norepinephrine and angiotensin II between baseline and 6-month follow-up (A) Systolic blood pressure. (B) Diastolic blood pressure. (C) Norepinephrine. (D) Angiotensin II. 
A

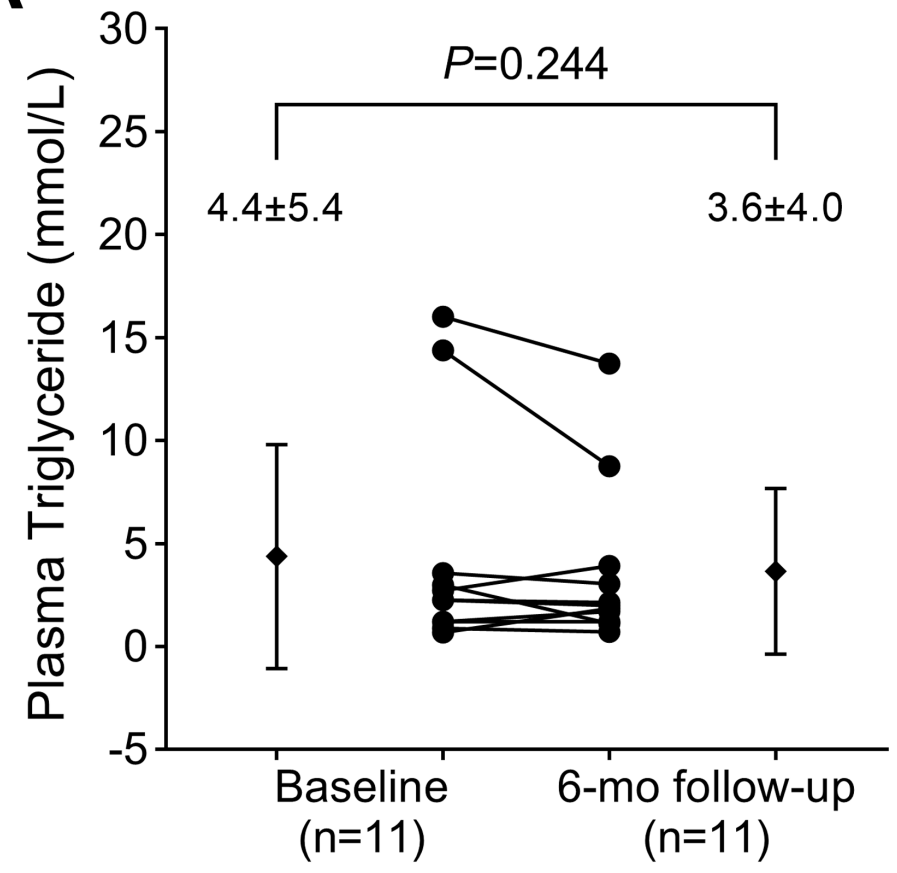

C

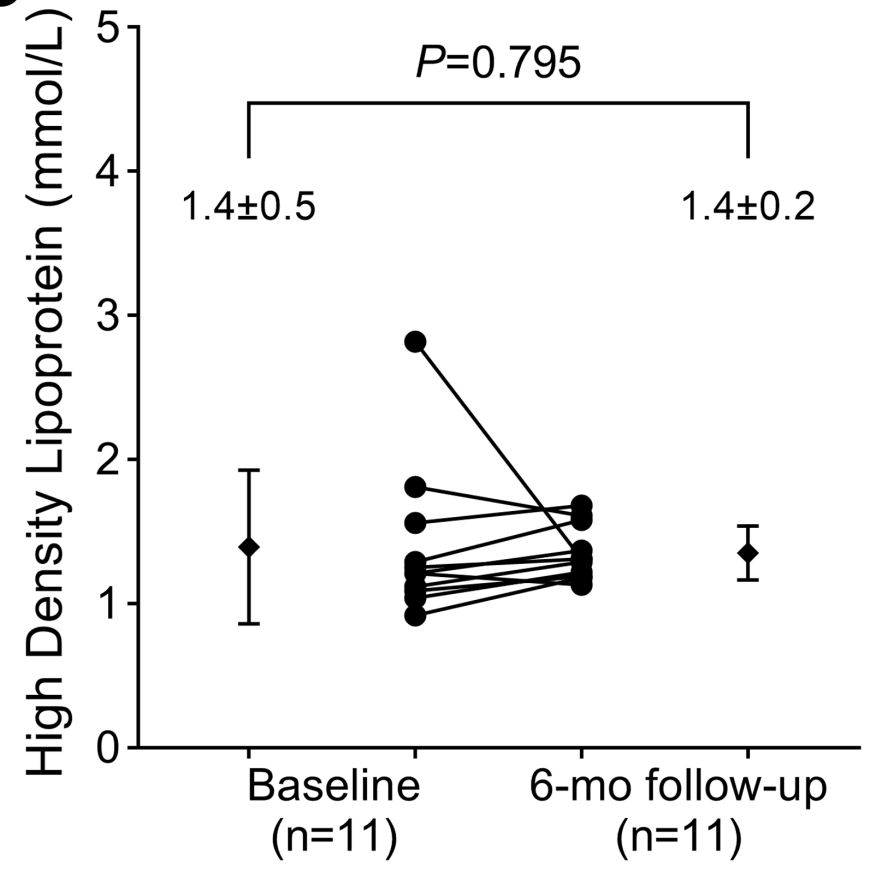

B

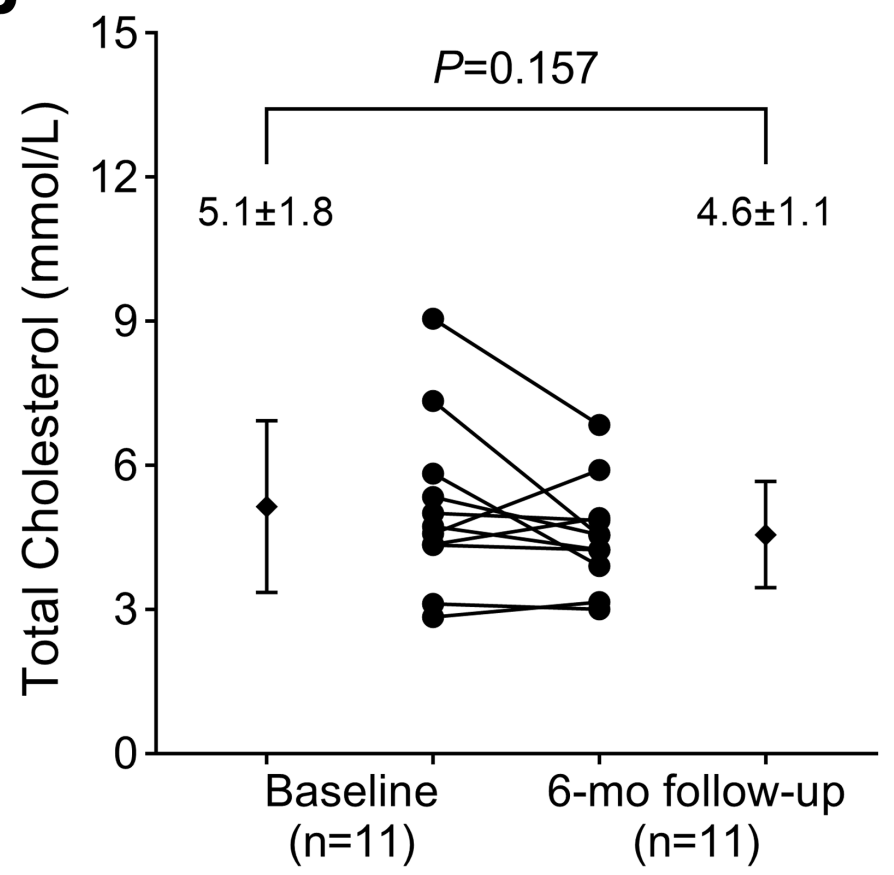

D

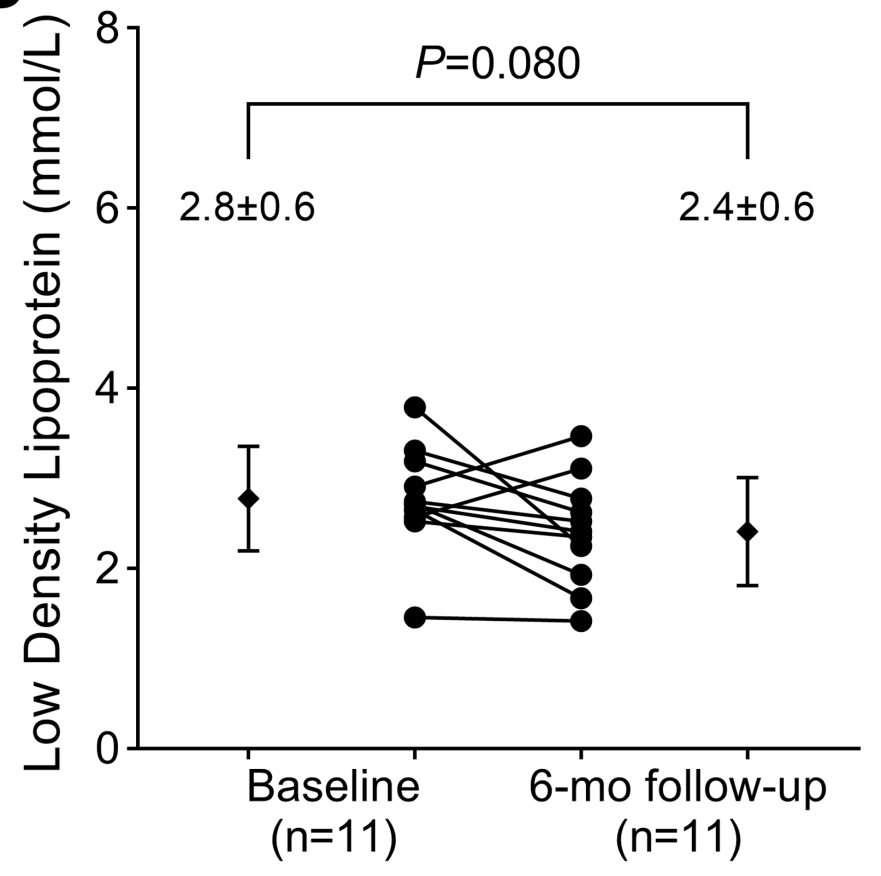

Figure 5

Changes of plasma lipids between baseline and 6-month follow-up. (A) Plasma triglyceride. (B) Total cholesterol. (C) High density lipoprotein. (D) Low density lipoprotein. 
A

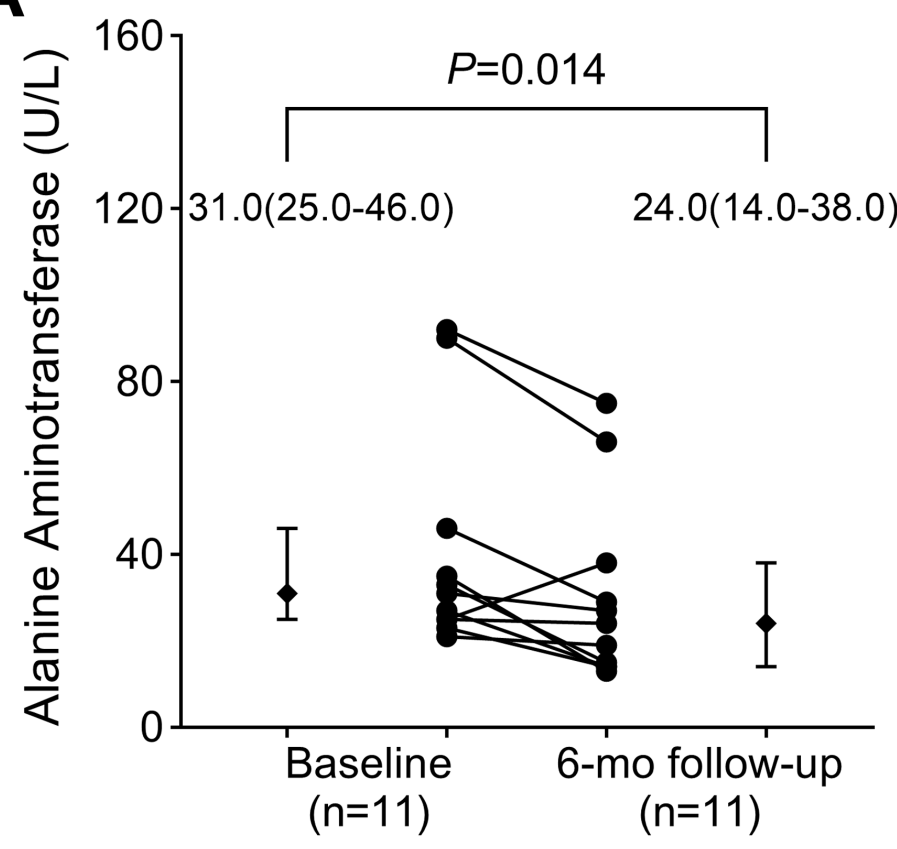

C

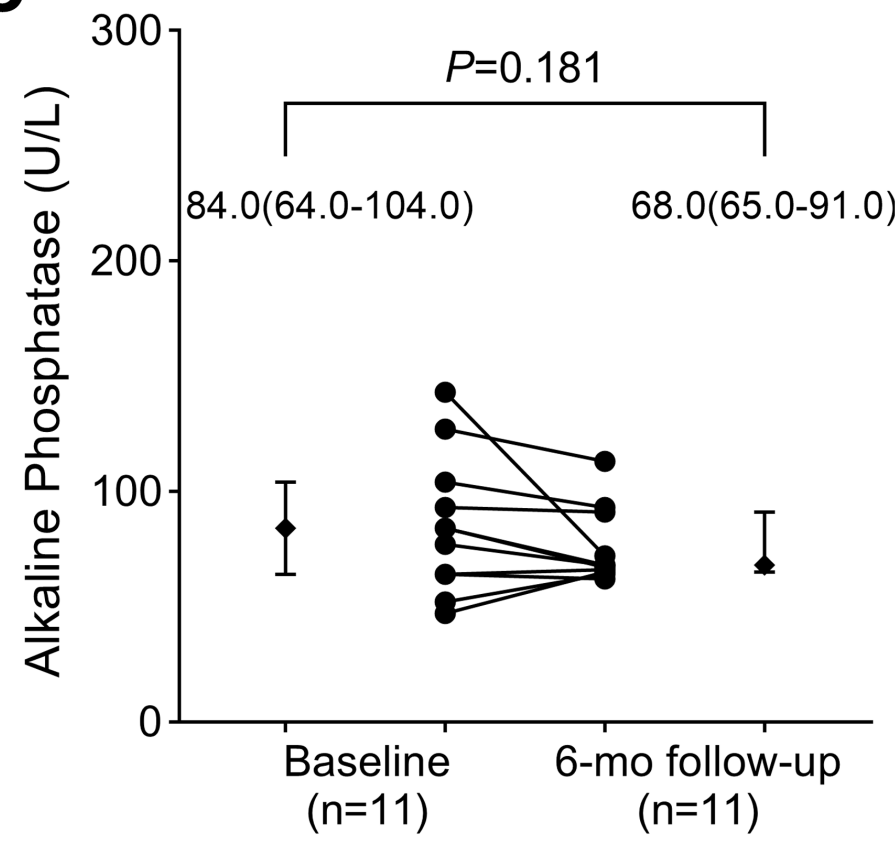

B

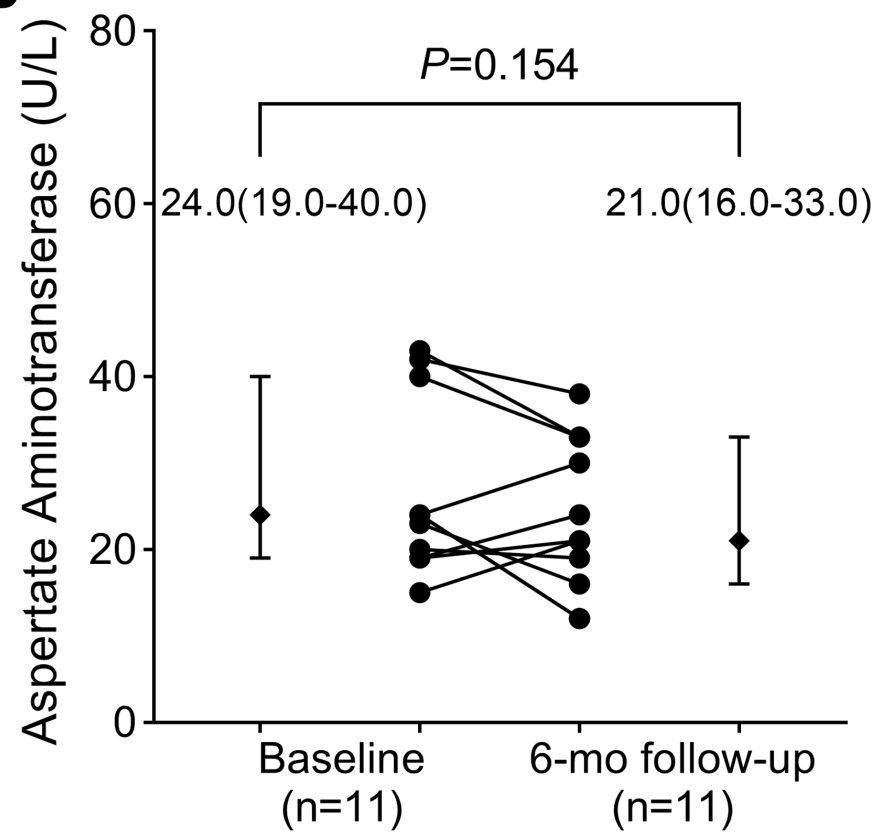

D

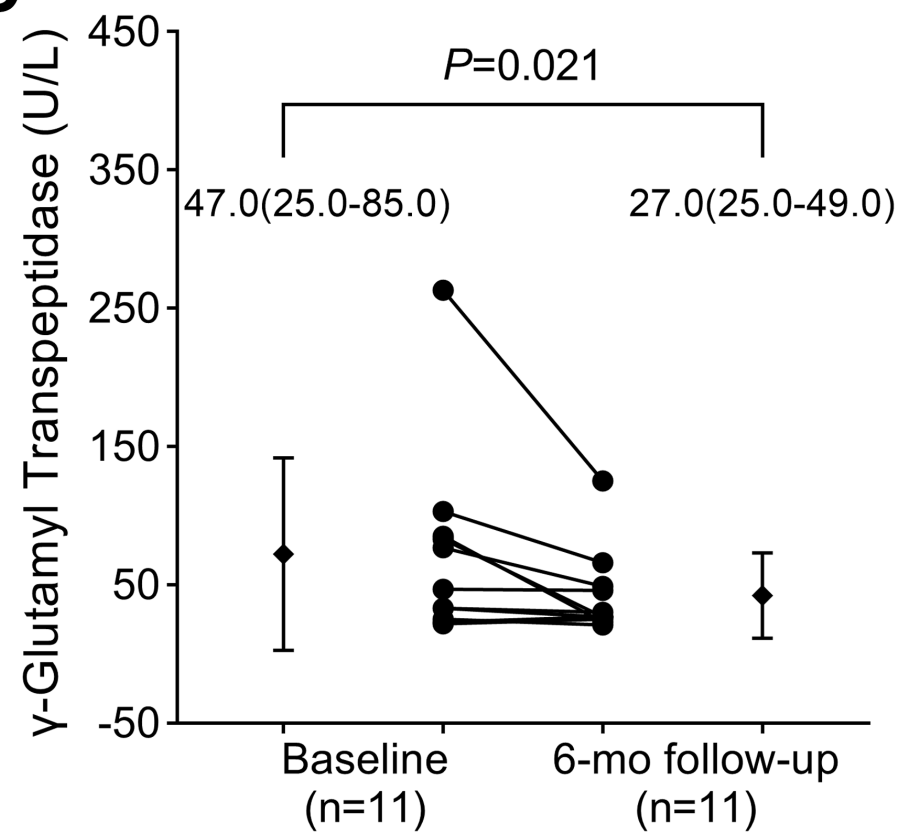

Figure 6

Changes in transferase between baseline and 6-month follow-up. (A) Alanine aminotransferase. (B) Aspertate aminotransferase. (C) Alkaline phosphatase. (D) Y-glutamyl transpeptidase. 